\title{
Le retornado dans le discours et les politiques des gouvernements de Rafael Correa (2007-2017)
}

Retour sur un tournant dans le traitement institutionnel de la question migratoire

\section{Amanda Bernal}

\section{(2) OpenEdition}

Journals

Édition électronique

URL : http://journals.openedition.org/agedor/5373

DOI : 10.4000/agedor.5373

ISSN : 2104-3353

Éditeur

Laboratoire LISAA

Référence électronique

Amanda Bernal, «Le retornado dans le discours et les politiques des gouvernements de Rafael Correa (2007-2017) », L'ÂAe d'or [En ligne], 12 | 2019, mis en ligne le 01 octobre 2020, consulté le 15 octobre 2020. URL : http://journals.openedition.org/agedor/5373 ; DOI : https://doi.org/10.4000/agedor.5373

Ce document a été généré automatiquement le 15 octobre 2020

L'Âge d'or. Images dans le monde ibérique et ibéricoaméricain 


\section{Le retornado dans le discours et les politiques des gouvernements de Rafael Correa (2007-2017)}

Retour sur un tournant dans le traitement institutionnel de la question migratoire

\section{Amanda Bernal}

\section{NOTE DE L'AUTEUR}

J'ai écrit cet article dans le cadre de ma thèse consacrée à la question du retour en Amazonie équatorienne. Il fait office d'une note de recherche qui doit être complétée par des données recueillies sur le terrain, afin de confronter la vision institutionnelle du retour au retour tel qu'il est vécu par les migrants.

Nous adressons à nos frères migrants l'expression de notre vive gratitude. Ce sont eux, ces exilés de la pauvreté, expulsés de leur propre terre, qui ont su porter la Patrie dans leur cœur [...]; ce sont eux qui nous ont nourris, par leurs peines, par leurs sacrifices, par leurs remises, malgré la

distance et le déracinement. Rentrez, chers frères, l'Équateur, votre pays, vous attend les bras et l'âme grands ouverts. ${ }^{1}$

Si Rafael Correa consacre de longues minutes de son discours d'investiture aux migrants, un groupe qui a été défini sous son mandat comme un groupe «d'attention prioritaire", c'est parce qu'il a choisi de faire de la question migratoire un axe fédérateur autour duquel il a construit son projet de Révolution citoyenne. En se positionnant comme celui qui marquerait une rupture avec la «longue et triste nuit 
néolibérale $»^{2}$, Rafael Correa a fait du retour, conçu comme l'acquittement d'une dette historique envers les " exilés de la pauvreté », une ligne directrice forte de sa politique.

Dès les premiers mois de son mandat, entamé en janvier 2007, le leader d'Alianza PAIS a en effet changé la façon dont l'Équateur se positionnait par rapport à sa diaspora, en lançant les premières mesures qui aboutiraient, en 2008, au Plan Bienvenid@s a casa. Avant lui, quelques mesures d'un transnationalisme mené par l'État avaient émergé, mais leur impact était resté marginal dans la sphère politique et administrative. Les politiques de lien avec la diaspora et plus spécifiquement les politiques du retour proposées sous la présidence de Rafael Correa se distinguent à la fois de celles initiées par ses prédécesseurs et de celles mises en place dans d'autres pays de la région tant par leur envergure comme par la place qu'elles ont prises sur la scène politique. C'est un retour conçu au sens large comme un retour économique, politique et culturel à la nation équatorienne qui fait la spécificité du cas équatorien, sur lequel je me pencherai dans cet article.

3 Je reviendrai, dans un premier temps, sur les flux migratoires successifs qui ont structuré le champ migratoire équatorien et sur les contextes dans lesquels ont eu lieu ces départs parfois massifs vers l'étranger. Je m'attacherai ensuite à analyser la place centrale qu'a pris le retour dans le projet de Revolución ciudadana. J'argue que qu'il est devenu le socle rhétorique sur lequel a été pensée la reconstruction de la patria, la preuve tangible d'une véritable rupture avec "longue et triste nuit néolibérale». Comme le rappelle Sayad, le "discours sur la réinsertion, indépendamment de ses effets, prend la forme [...] d'une revanche sur l'histoire ancienne [...] il se veut comme une manière magique de nier cette histoire en en niant les effets ou en les réintégrant " ${ }^{3}$. Le retour des migrants a ainsi été présenté comme une preuve de la renaissance de la nation équatorienne, puisqu'il s'agissait finalement d'un retour à la coïncidence entre 《identités ", «frontières » et "ordres », c'est-à-dire à la triade conceptuelle que Vertovec place au cœur de la construction de l'imaginaire national et que l'émigration massive de la fin des années 1990 avait mise à mal.

4 Après avoir étudié le caractère instrumental de cette rhétorique du retour dans la reconstruction de l'équatorianité, j'interrogerai un décalage sémantique qui me paraît crucial pour comprendre l'inadéquation entre la façon dont la sphère institutionnelle a pensé le retour et l'expérience de la plupart retornados. Alors que le retour à la casa tel qu'il est construit dans les discours de Correa et dans le Plan Bienvenid@s a casa implique un retour physique au territoire équatorien et un retour symbolique à la nation équatorienne, la casa que construisent les migrants est ancrée dans des lieux donnés (dans le pays d'immigration comme d'émigration) et dans des liens sociaux transnationaux qui n'ont pas attendu la médiation de l'État pour se développer. C'est là que la vision réductrice de l'émigration comme déracinement, qui domine dans le discours présidentiel, trouve ses limites : les migrants habitent différents lieux au cours de leur mobilité, et leurs lieux d'ancrage se recomposent en fonction de trajectoires plurielles qui ne correspondent pas toujours à la vision monolithique du retour qui sous-tend le Plan Bienvenid@s a casa. Je complèterai cette analyse discursive d'une étude de l'évolution des programmes inclus dans le Plan et de leur réception. Je chercherai notamment à déconstruire la conception du retornado comme agent économique qui $\mathrm{y}$ domine, en la confrontant à la pluralité des expériences des migrants. 


\section{Entre crisis y crisis : une brève description de la structuration du champ migratoire équatorien}

5 Si Rafael Correa rappelle de façon récurrente qu'avant la fin des années 1990, l'Équateur n'était pas un pays d'émigration, force est de constater que les filières de la migration équatorienne avaient déjà commencé à se structurer des décennies auparavant. C'est dès la fin des années 1960 que s'établissent des chaînes migratoires reliant dans un premier temps les zones rurales et les petites villes de l'Austro équatorien aux États Unis, principalement aux villes de New York et Chicago ${ }^{6}$.

6 À la fin des années 1990, on constate toutefois un tournant: on assiste à une massification de l'émigration, puisque qu'environ $10 \%$ de la population totale quitte le pays entre la fin des années 1990 et le début des années $2000^{7}$. On observe également une diversification des lieux tant de destination que d'origine des migrants: les Équatoriens émigrent de plus en plus vers les pays du sud de l'Europe, en particulier vers l'Espagne, et ils ne partent plus seulement des Andes australes. Les autres provinces andines et les régions côtières sont tout aussi touchées par le phénomène migratoire pendant cette décennie. Ces nouveaux départs se distinguent également des flux précédents du fait d'une forte présence de femmes qui deviennent les «têtes de pont» des réseaux sociaux transnationaux qui structurent le champ migratoire équatorien. Il s'agit généralement de migrants et de migrantes partis de milieux urbains, et qui sont en moyenne plus qualifiés que ceux venant des zones rurales de l'Austro ${ }^{8}$.

7 Même si l'Organisation internationale pour les migrations (OIM) définirait la plus grande partie de ces migrants comme des sujets d'une migration économique et non d'une migration forcée', Rafael Correa emploie un vocabulaire relevant principalement de la seconde catégorie. Ces migrants sont selon lui des victimes d'une « expulsion » : ce sont des « exilés de la pauvreté » et non des agents en quête de meilleures opportunités pour eux-mêmes et pour leurs familles. Pour comprendre le champ lexical qu'il mobilise, il faut en revenir au contexte dans lequel s'est produite cette massification de l'émigration, que les chercheurs ont appelé la estampida migratoria ecuatoriana ${ }^{10}$. La fin des années 1990 est en effet marquée par une grave crise économique et socio-politique qu'il est nécessaire de prendre en compte. Aux scandales de corruption et à l'instabilité politique qui en ont découlé se sont ajoutés un effondrement du système bancaire et une profonde récession économique, à laquelle le gouvernement a répondu par une dollarisation très fortement contestée.

Si bien des migrants avec lesquels j'ai eu l'occasion de discuter parlent explicitement de la crise comme un des facteurs qui les ont poussés à quitter leur pays et souvent leurs familles, Rafael Correa va beaucoup plus loin. En établissant une causalité directe entre crise et émigration, le leader d'Alianza PAIS efface l'agency des migrants et ne tient aucunement compte des stratégies que les Équatoriens ont pu déployer pour faire face aux contraintes structurelles ${ }^{11}$. Son explication mono-causale est bien sûr réductrice, et d'autres facteurs sont à prendre en compte, tant à l'échelle individuelle que collective. Alba Goicoechea et Franklin Ramírez ont justement cherché à remettre en question cette prégnance des facteurs structurels dans la vague d'émigration des années 1990 : à travers leur étude, ils ont mis en évidence l'importance de l'imaginaire collectif dans le processus de prise de décision des migrants ${ }^{12}$. Pour eux, la forte émigration de la fin du siècle a une double racine : elle s'explique du côté push par une crise de représentativité 
politique, nourrie par un manque de confiance envers des institutions démocratiques incapables d'assurer la reproduction sociale d'une grande partie de la population ; et du côté pull par une construction des lieux de destination dominée par l'illusion.

J'ai moi-même constaté l'importance de ces facteurs relevant des représentations lors de mon travail de terrain pour mon master 2, effectué à Londres. J'y ai travaillé sur les processus de ré-émigration des migrants latino-américains ayant fui la crise espagnole pour s'installer dans la capitale britannique, et j'ai eu l'occasion d'échanger avec de nombreux Équatoriens et Équatoriennes partis à cette période. C'est lors d'un entretien avec deux femmes originaires de Gualaquiza, une petite ville située dans la province amazonienne de Morona Santiago, que ces questions ont surgi de façon particulièrement significative. Je leur ai en effet demandé si beaucoup de leurs connaissances étaient parties en même temps qu'elles, ce à quoi elles ont répondu en riant qu'elles avaient retrouvé tout Gualaquiza à Madrid. Quand je les ai questionnées sur les raisons qui avaient provoqué ces départs massifs, voici ce que l'une d'entre elles m'a dit :

Il y en a un qui est arrivé, et ensuite, comme ça, tout le monde a commencé à partir. La facilité, c'était que pour l'Espagne, on n'avait pas besoin de visa, pour les ÉtatsUnis si. En Espagne, on entrait comme touristes [...] Comme les gens voyaient qu'il y en avait un qui réussissait à passer, l'autre aussi, eh bien tout le monde disait : » Ah, qu'en Espagne on se fait des sous, qu'en Espagne tout... ». Alors, tout le monde s'est ouvert les yeux, et tout le monde est parti. ${ }^{13}$

10 Ces deux facteurs, relevant de l'imaginaire bien plus que des contraintes structurelles, ont fait que beaucoup d'Équatoriens ont été incapables de projeter leur biographie personnelle dans l'espace national. En avançant de tels arguments, Alba Goicoechea et Franklin Ramírez nuancent la position de Rafael Correa, pour qui l'émigration est une simple réponse à la détérioration des conditions économiques. Ils insistent sur l'importance des constructions sociales et culturelles au sens large dans le processus de prise de décision des migrants.

11 Un peu moins d'une dizaine d'années après cette crise équatorienne - à partir de 2008 les migrants subissent les conséquences d'une autre crise économique, dans les pays de destination cette fois. Leur vulnérabilité dans le contexte de récession économique s'explique par une multiplicité de facteurs, parmi lesquels figurent la fragilité de leur statut légal, leur concentration dans des secteurs non-compétitifs et peu qualifiés du marché du travail - citons à titre d'exemple le secteur de la construction, dans lequel était employé un très grand nombre des Équatoriens installés en Espagne - et finalement leur taux d'endettement souvent élevé.

12 Face à la crise, les ménages migrants ont vite déployé des stratégies de mobilité à la fois professionnelle, généralement descendante, et géographique - interne ou internationale. Ceux qui ont recouru à ce deuxième type de mobilité ont quitté le pays dans lequel ils s'étaient installés pour d'autres destinations : c'est le cas de beaucoup d'Équatoriens résidant en Espagne, qui sont partis pour se fixer au Royaume-Uni ou aux États-Unis. Il faut préciser que ces nouveaux départs ont été grandement facilités par le statut légal de la plupart de ces Équatoriens, naturalisés au cours des vagues de régularisation extraordinaire organisées par le gouvernement espagnol. Un certain nombre d'Équatoriens ont en revanche décidé d'une mobilité de retour. Ces retours n'ont pas été massifs, mais il ne faut pas les négliger : selon le dernier recensement, 63888 migrants étaient rentrés au pays entre 2005 et $2010^{14}$. Il faut cependant prendre un peu de recul par rapport à ce chiffre : il ne s'est écoulé que deux ans entre le début 
de la Gran Recesión et le recensement, deux ans pendant lesquels les familles migrantes ont essayé d'autres stratégies de mobilité - professionnelle et géographique interne en général - avant d'opter pour la solution du retourlesquels ont été plus nombreux les années suivant le recensement.

La crise a touché la population migrante équatorienne, mais également d'autres communautés latino-américaines vivant à l'étranger. Cela explique pourquoi on a assisté à une multiplication de programmes émanant des gouvernements des pays d'émigration de la région prônant un retour conçu comme volontaire : le Pérou a mis en place des politiques dirigées vers la diaspora péruvienne, appelée le Quinto Suyo (la Cinquième Région - conçue comme une extension des quatre régions qui formaient l'Empire inca ou Tawantinsuyu) dès 2005, la Colombie a quant à elle mis en place le Plan de Retorno Positivo en 2009, et en Bolivie le Plan de Retorno y Reintegración Sostenible para migrante bolivianos y bolivianas est apparu deux ans après, pour ne citer que quelques voisins andins ${ }^{15}$. Ces programmes sont généralement vus comme des réponses à la conjoncture internationale, tout comme au durcissement des politiques migratoires dans les pays d'accueil, mais le cas de l'Équateur se distingue de celui des autres pays de la région du fait que l'apparition du Plan Bienvenid@s a casa est due avant tout à des changements politiques à l'intérieur du pays ${ }^{16}$. Comme l'explique Ana Margheritis dans son étude sur les paradoxes du transnationalisme mené par l'État en Équateur, le développement des politiques de mise en lien avec la diaspora est à comprendre comme «l'étendard» d'un mouvement de réformes plus globales dans la politique intérieure.

14 Je chercherai donc à replacer les politiques du retour mises en place sous les gouvernements de Rafael Correa à l'intérieur d'un tournant politique plus large qui s'est opéré à partir de sa première élection en 2006. Pour ce faire, je mettrai en évidence le basculement discursif dans la façon dont l'État équatorien envisageait jusque-là ses rapports à la diaspora et j'analyserai le rôle de ce basculement dans le projet politique de Revolución ciudadana porté par Rafael Correa. Je me pencherai en dernier lieu sur l'écart entre les discours, les textes normatifs et les politiques mises en place.

\section{Des balbutiements d'un transnationalisme mené par l'État au tournant des élections de 2006 : repenser les liens entre État et diaspora équatorienne}

15 Les premières mesures de ce que les chercheurs anglophones ont appelé State-led transnationalism (transnationalisme mené par l'État) apparaissent dès les années 1990, avant même l'estampida migratoria. Le transnationalisme envisagé sous cet angle désigne «l'ensemble des politiques et des programmes nationaux institutionnalisés qui cherchent à étendre la portée du pouvoir de régulation politique, économique et social de l'État-nation, afin d'inclure les émigrants et leurs descendants qui se trouvent en dehors du territoire national $\aleph^{17}$. Cette extension du pouvoir de régulation de l'Étatnation peut passer par divers canaux, parmi lesquels se trouvent la création d'institutions spécifiques destinées à gérer les besoins et à répondre aux demandes des émigrants, la mise en place de mécanismes de canalisation des remises, l'extension des droits politiques des citoyens résidant à l'étranger, pour n'en citer que quelques-uns. 
16 En Équateur, l'État commence à véritablement s'intéresser à ses citoyens vivant à l'étranger des décennies après la première vague importante d'émigration vers les États-Unis. Une multiplicité de facteurs explique pourquoi les gouvernements au pouvoir commencent à prendre des mesures destinées à donner une plus grande reconnaissance aux populations migrantes. Une pression se fait sentir du côté de la société civile, puisque les associations de migrants équatoriens s'organisent et militent, notamment pour l'obtention d'une extension de leurs droits politiques ${ }^{18}$. Ces revendications font écho à un contexte international plus large qui met en avant la question des droits des migrants et pousse l'État équatorien à se positionner sur ces questions. Il ne faut pas non plus négliger les facteurs économiques : n'oublions pas que les remises deviennent une source de devises étrangères de plus en plus importantes (elles deviendront, dans les années 2000, la seconde source de devises étrangères après le pétrole ${ }^{19}$ ). Les gouvernements successifs ont donc tout intérêt à encourager ces remises et à les canaliser, ce qui passe nécessairement par une réponse aux besoins exprimés par cette part toujours grandissante d'Équatoriens vivant en dehors du territoire national, dans ce que Rafael Correa appellera plus tard la Quinta Región (la Cinquième Région). C'est ainsi que la double nationalité est accordée aux émigrants la demandant dès 1994. Le droit de vote pour les élections présidentielles est quant à lui inscrit dans la Constitution de $1998^{20}$, mais il faudra attendre huit ans pour que les migrants puissent l'exercer. Quant à la protection qu'est censée garantir l'État, aucun mécanisme n'est mis en place pour la permettre réellement - c'est une des grandes limites de la période.

17 Ce n'est qu'à partir de l'an 2000, et en raison de la pression croissante venant de plusieurs franges de la société civile que l'accent est effectivement mis sur le droit des migrants et les politiques de lien avec la diaspora. L'année suivante, une Direction générale de soutien aux Équatoriens à l'étranger est créée au sein du ministère des Affaires étrangères. Il s'agit du premier corps institutionnel entièrement dévoué à la diaspora équatorienne. C'est également en 2001 qu'est élaboré le Plan national des migrants équatoriens à l'étranger, dont les trois principaux objectifs étaient d'assurer la protection des migrants à l'étranger, d'assister les familles des migrants restées au pays, et d'établir des relations plus étroites avec les principaux pays vers lesquels avaient émigré les Équatoriens. Le fait de travailler tant avec les migrants qu'avec leurs proches part d'une volonté de traiter du fait migratoire et de ses conséquences dans leur intégralité. Le migrant n'apparaît donc pas comme un déraciné - une image que Rafael Correa mobilisera de façon récurrente quelques années après - mais comme un individu qui a gardé et cultivé les liens qui l'unissent à sa famille restée sur le territoire équatorien. Cependant, comme le montre très bien William Herrera Ríos ${ }^{21}$ dans sa thèse consacrée à la question du retour sous un prisme institutionnel, ce plan n'a jamais donné lieu à de véritables politiques, il est resté un simple document de travail. L'année suivante, en 2002, est présenté un autre programme qui reste lettre morte. Ce Programme d'aide, d'épargne et d'investissement pour les migrants équatoriens et les membres de leur famille, destiné à canaliser les remises de façon plus efficace et à conseiller les migrants dans les investissements qu'ils font dans leur pays d'origine ne se concrétisera jamais. Il faudra attendre quelques années pour que ce passage du discours et des textes aux politiques appliquées s'opère. marginalisées dans la sphère politique et bureaucratique. Ce n'est que lors de ces 
élections que les migrants exercent effectivement leur droit de vote pour la première fois, mais les résultats reflètent un désenchantement persistant par rapport à la política : peu de gens s'inscrivent sur les listes électorales à l'étranger, et parmi les inscrits, le taux d'abstention atteint presque les $40 \%$. S'ils représentent plus de $10 \%$ de la population, les voix des migrants ne correspondent finalement qu'à $1,5 \%$ du total des voix $^{22}$. Dans les circonscriptions de l'étranger, les votes nuls et blancs l'emportent sur le nombre de voix obtenues par Noboa, qui finit en tête alors qu'il ne propose aucune action spécifique concernant les Équatoriens à l'étranger dans son plan de gouvernement ${ }^{23}$.

Si l'on analyse d'un peu plus près les programmes politiques des candidats, on se rend compte que seuls 4 des 13 candidats ${ }^{24}$ abordent la question des droits des migrants, parfois de façon tout à fait superficielle. C'est le programme de Rafael Correa - qui n'arrive pourtant pas en tête dans les circonscriptions de l'étranger - qui développe le plus la question migratoire, d'un point de vue politique, économique et social. Je ne m'y arrêterai pas en détail, puisque j'aurais l'occasion de revenir sur les politiques effectivement mises en place au cours de ses mandats dans la dernière partie de mon étude, mais j'aimerais souligner ici le rôle instrumental qu'a joué la question migratoire dans l'élection de Rafael Correa.

20 Le leader d'Alianza PAIS a en effet su en faire une véritable ressource politique, comme le montre très bien Ana Margheritis dans l'étude détaillée qu'elle fait du rôle clef qu'il a pu avoir dans l'arrivée de Rafael Correa au pouvoir ${ }^{25}$. En donnant une véritable place aux Équatoriens de l'extérieur dans son projet politique - chose qui n'avait jamais été faite auparavant - il a pu fédérer une coalition sans véritable identité politique autour d'une question symbolique et qui touchait directement une grande partie des électeurs, dans la plupart des provinces de l'Équateur. Les références récurrentes à l'émigration massive de la fin des années 1990 lui ont également permis de se positionner contre ses opposants politiques, puisqu'il a construit la croissance spectaculaire des flux migratoires comme une véritable "tragédie nationale ", une conséquence directe des réformes néolibérales imposées par les gouvernements précédents. Enfin, cela lui a permis de faire de la question migratoire l'« étendard " d'un projet de refonte de la nation équatorienne, basé sur un retour des " exilés de la pauvreté ». Ce retour est conçu comme un retour physique vers le territoire équatorien, mais aussi et surtout comme un retour symbolique à la nation équatorienne. Voici la mission que se proposait le Secrétariat national du Migrant (SENAMI) avant que ses fonctions ne soient reprises par le ministère des Affaires étrangères et de la mobilité humaine : «Nous défendons un retour au sens large: un retour qui n'implique pas nécessairement un retour physique, mais plus généralement, une récupération des capacités. $»^{26}$

21 Ce projet inclusif de refonte de la nation se structure autour d'une resignification de ce qu'est la communauté nationale, visible dans quatre tournants discursifs majeurs. Je reprendrai ici, en les développant, certains des arguments avancés par Paolo Boccagni dans son étude de la construction de cette Cinquième 'égion de l'Équateur ${ }^{27}$.

Cette refonte se manifeste d'abord dans une renaturalisation de l'appartenance des migrants à la nation équatorienne, renaturalisation qui passe par la mobilisation de métaphores de l'enracinement et de la parenté. Comme le rappelle Lisa Malkki ${ }^{28}$, les métaphores de type botanique sont extrêmement récurrentes dans la rhétorique de construction de la communauté imaginée qu'est la nation, puisqu'elles permettent de naturaliser le lien entre des personnes et des lieux, chaque nation étant présentée 
comme un gigantesque arbre généalogique, enraciné dans le sol qui le nourrit. En parlant de l'émigration comme d'un "déracinement", Rafael Correa rappelle non seulement que les migrants sont rattachés à leur tierra par un lien organique, mais il présente aussi leur départ comme un déchirement qui ne pourra se résoudre que par un retour à l'état naturel qu'est la résidence sur le territoire national. L'autre métaphore qui surgit de façon récurrente dans ses discours est celle de la parenté : le leader d'Alianza PAIS parle constamment des migrants comme des « frères » qui ont dû quitter leurs familles, tout comme la grande famille imaginée qu'est la nation équatorienne, malgré eux. Alors que l'équatorianité a traditionnellement plutôt été pensée comme confrontation à l'altérité - que ce soit sur le plan territorial, dans la confrontation avec l'expansionnisme péruvien, ou sur le plan ethnique, dans le dépassement de ce qui a été construit comme le "problème indigène " par la création d'une nouvelle « race » mestiza ${ }^{29}$-, elle est maintenant pensée comme un appel à l'unité, qui passe par la mobilisation d'une image simple : celle de la famille. Comme le fait très justement remarquer Paolo Boccagni, celle-ci «a été instrumentale dans un discours politique qui célèbre ce qu'ont en commun ceux qui sont restés et ceux qui sont partis, tout en minimisant les divisions raciales et ethniques encore très importantes dans la société nationale $»^{30}$.

La resignification des frontières de la communauté nationale passe aussi par une extension métaphorique du territoire. C'est à cette extension que l'on doit l'apparition d'une Quinta Región (Cinquième Région), qui vient s'ajouter aux quatre régions préexistantes que sont la Costa (la Côte), la Sierra (les Andes), l'Oriente (l'Amazonie) et la région insulaire (les Galapagos). Cette extension métaphorique permet de dépasser le décalage produit par la migration entre citoyenneté et résidence, et constitue le second tournant discursif majeur.

24 Rafael Correa se démarque également de ses prédécesseurs en donnant aux migrants (résidant à l'extérieur ou de retour) un rôle moteur dans son projet de Revolución Ciudadana. Il en fait les nouveaux acteurs clefs du développement du pays, dotés du capital économique et humain nécessaire pour faire prospérer l'économie nationale. Cela est loin d'être évident, puisque nombreux sont ceux pour qui le retour a d'abord été une façon de résister à une situation conjoncturelle qui ne leur permettait plus de vivre correctement dans les pays dans lesquels ils s'étaient installés : comment l'État se positionne-t-il alors face à ces migrants de retour qui ne rentrent pas dans le moule du retornado volontaire et capable de mobiliser différentes ressources accumulées en migration? C'est là que le décalage entre vision institutionnelle et expériences du retour devient manifeste, comme nous aurons l'occasion de le voir de façon plus détaillée dans la partie suivante.

Cette refonte de l'équatorianité passe finalement par une reconnaissance des migrants comme sujets de droit, au même titre que n'importe quel Équatorien et plus largement citoyen du monde. Je le précise en rappelant que le leader d'Alianza PAIS se fait le porteur d'un projet de citoyenneté universelle qui entre nécessairement en tension avec d'autres points de son programme. Il est bien sûr paradoxal que quelqu'un qui insiste tant sur le retour en force de la nation équatorienne et a fait de la valorisation $\mathrm{du}$ fait d'être et de se sentir équatorien un des objectifs de sa politique migratoire, se positionne comme le chantre d'un projet de citoyenneté universelle.

Ces tournants discursifs se traduisent en un certain nombre de politiques de lien avec la diaspora auxquelles je consacrerai la dernière partie de cette étude. J'y mettrai 
notamment en relief le basculement d'une priorité donnée au droit à une priorité donnée au développement qui se joue dans le passage du discours aux politiques. Je chercherai ainsi à montrer le décalage qui se crée entre la figure univoque du retornado comme agent économique puis comme «cerveau » qui domine dans les programmes mis en place, et les expériences et trajectoires multiples de ceux qui entreprennent le voyage du retour.

\section{Mise en place des politiques du retour et réception : les limites d'un projet « révolutionnaire " ${ }^{31}$}

27 Les documents ${ }^{32}$ qui sont pensés dans les premières années du gouvernement de Rafael Correa et qui fixent les idées qui régissent ce tournant dans les politiques migratoires reposent sur la rhétorique de défense des droits des migrants que j'ai évoquée dans la partie précédente, rhétorique qui sera inscrite dans la Constitution de Montecristi en 2008. La politique migratoire mise en avant par le nouveau gouvernement se fixe ainsi trois objectifs principaux : la garantie du droit d'émigrer, du droit de rester, et du droit de rentrer " de manière volontaire, digne et durable $»^{33}$. Ce sont ces trois impératifs que je chercherai à questionner dans cette dernière partie.

28 Les images et le lexique mobilisés par la SENAMI dans les brochures de présentation du Plan de Retorno Voluntario, Digno y Sostenible, "Bienvenid@s a casa" prolongent la rhétorique déployée par Rafael Correa autour de la question du retour : l'émigration est vue comme exil, un déracinement auquel seul le retour à la casa, entendu comme un retour au territoire et à la nation équatorienne, peuvent remédier. En plus d'ôter tout agency aux migrants, en faisant d'eux les simples victimes de la mauvaise gouvernance des dirigeants précédents, cette rhétorique oublie que les migrants n'ont pas laissé leur casa en Équateur, mais que la casa comme lieu géographique et comme lieu métaphorique d'attachement et d'identification personnelle, est reconstruite et renégociée au cours des migrations, tant dans ses aspects matériels que symboliques ${ }^{34}$.

Il y a donc à la fois un contraste de nature et d'échelle entre la casa telle qu'elle est construite dans la sphère institutionnelle et celle qu'habitent réellement les retornados ${ }^{35}$. Le chez-soi que construisent les migrants, à l'étranger comme à leur retour, est une véritable translocalitée ${ }^{36}$, c'est-à-dire le point d'articulation d'une multitude de réseaux transnationaux qui permettent aux migrants de rester ancrés ici et là-bas. Si l'on reprend la terminologie d'Henri Lefebvre ${ }^{37}$, cela signifie que l'« espace vécu» des migrants - celui des représentations et de l'imaginaire - comprend non seulement le lieu qu'ils habitent, mais les différents lieux dans lesquels ils ont pris place au cours de leur trajectoire de mobilité. La casa telle qu'elle est postulée par les institutions qui doivent prendre en charge la question migratoire est bien différente : c'est un lieu au sens plus traditionnel du terme, c'est-à-dire le support d'une identité stable s'appuyant sur un enracinement durable dans une localité aux frontières bien définies. À ce contraste de nature vient s'ajouter un contraste d'échelles entre la casa conçue et la casa vécue, puisqu'il y a un décalage entre la casa projetée par le gouvernement, qui s'étend à tout le territoire national, et la casa que construisent et reconstruisent les migrants, dont les contours sont matériellement définis, tout en pouvant être multiples, et qui repose sur des liens interpersonnels dont la réalité est beaucoup plus tangible que la communauté imaginée qu'est la nation. 

décalages entre les politiques proposées aux retornados et la réalité vécue par ces migrants. Pour les comprendre, je reviendrai d'abord brièvement sur les différentes politiques mises en place au moment de l'apparition du Plan Bienvenid@s a casa ${ }^{38}$. cet ensemble de politiques. Il avait pour objectif principal la mise en relation des différents acteurs impliqués dans le processus migratoire. Il visait à faciliter la création et le maintien des liens sociaux transnationaux à travers la mise à disposition d'une plateforme virtuelle qui se voulait être un espace de communication et d'échange entre migrants, non-migrants et institutions chargées des questions migratoires. Bien qu'il ne soit plus consultable depuis la disparition de la SENAMI, c'était un portail où l'on trouvait aussi bien des informations d'ordre institutionnel se rapportant aux politiques mises en place et aux services proposés par les structures gouvernementales implantées à l'étranger, qu'un espace de partage plus personnel. Les migrants pouvaient en effet parler de leur vécu à travers différents forums, ou se servir du chat pour communiquer avec leur famille et leurs amis restés au pays. La plateforme recensait également les différentes associations de migrants équatoriens réparties de par le monde. Ce site devait donc permettre une mise en réseau à diverses échelles, allant de l'individuel à l'institutionnel en passant par des collectifs de la société civile.

Le Programa de Incentivos y Orientación a Personas Migrantes para la Inversión Social y Productiva (Programme d'Incitation et d'Orientation pour l'Investissement Social et Productif des Personnes Migrantes) - un second axe du Plan Bienvenid@s a casa regroupe un ensemble de mesures de canalisation des remises et de promotion des investissements productifs en Équateur. L'initiative la plus connue, et certainement la plus étudiée, a été le programme $E l$ Cucayo ${ }^{39}$. Il s'agissait d'un fond mis à disposition par l'État et qui permettait à des entrepreneurs dont le projet avait été sélectionné d'accéder à un capital d'amorçage non-remboursable couvrant en moyenne un peu plus de $20 \%$ de l'apport nécessaire pour la mise en place de l'activité, selon les chiffres de María Isabel Moncayo, qui a étudié le projet entre 2008 et $2010^{40}$. Ce programme, centré sur l'intégration économique des retornados a cependant eu une portée très limitée, mise en avant par de nombreux chercheurs. Entre 2008 et 2012, 301 personnes ont directement bénéficié du programme, et il faut souligner leur profil très particulier : elles avaient nécessairement le capital économique pour pouvoir monter un projet considéré comme " productif » par l'institution chargée d'attribuer les capitaux ${ }^{41}$.

Le troisième et dernier axe du Plan concerne le retour tel qu'on l'imagine plus communément : il s'agit d'un programme d'accompagnement au retour physique, aussi appelé Programa Volver a Casa (Rentrer à la maison). Celui-ci englobe trois mesures, destinées à des publics différents. La première mesure, ouverte à tous les Équatoriens souhaitant rentrer, consiste en une exonération d'impôts visant à faciliter le déménagement de divers objets et appareils destinés à un usage domestique, ainsi que de véhicules et d'outils de travail - dans des limites très clairement fixées. La SENAMI a également mis en place une aide financière ponctuelle proposée spécifiquement aux personnes considérées comme "vulnérables » (de par leur situation légale, économique ou sociale). Cette aide devait leur permettre de rentrer en Équateur de façon sécurisée, mais s'arrêtait au moment où les migrants arrivaient sur le sol équatorien. Aucun suivi de leur situation sur le moyen ou le long terme n'a été envisagé dans le cadre de cette politique. Enfin, les retours post-mortem ont également été envisagés, en réponse à une 
très forte demande de la part de la population migrante : tout un pan du programme Volver a Casa est ainsi dédié au rapatriement des corps des Équatoriens décédés à l'étranger.

Bien que ces programmes découlent d'une conception élargie du retour, puisqu'ils vont au-delà d'un simple accompagnement au retour physique et prennent en compte un retour plus symbolique, j'argue que les institutions chargées de les penser ont oublié que le retour physique est bien plus qu'un simple déplacement spatial vers le pays d'origine. Il s'agit d'un véritable processus de (ré) insertion économique, mais aussi sociale et culturelle, qui pose parfois les mêmes difficultés qu'une nouvelle migration, notamment pour ceux qui choisissent de rentrer en Équateur, mais de s'installer autre part que dans leurs communautés d'origine. C'est souvent le cas pour les migrants partis de zones rurales ou de petites villes, qui considèrent que la structure d'opportunités sera meilleure dans de plus grandes villes, et "rentrent» pour s'installer dans un lieu qui leur est finalement tout aussi peu familier que Madrid ou New York ${ }^{42}$. Les seuls migrants qui peuvent profiter d'une aide et d'un suivi du gouvernement sur le plus long terme - et ce sur un plan purement économique - sont les migrants possédant des ressources tangibles ou intangibles, c'est-à-dire le capital financier et humain nécessaire pour participer au développement de l'Équateur.

Les politiques d'assistance au retour ont d'ailleurs tendu à devenir de plus en plus exclusives avec le temps. On constate en effet un passage de politiques favorisant des entrepreneurs rentrés avec un petit capital financier - ceux pour qui le programme $\mathrm{El}$ Cucayo avait été pensé - à des politiques cherchant à attirer des migrants beaucoup plus qualifiés ${ }^{43}$. Le contenu du Plan Bienvenid@s a Casa change en effet à partir de 2011, au moment où est mis en place le programme Prometeo "Viejos Sabios". Celui-ci est destiné aux chercheurs étrangers, mais aussi aux chercheurs équatoriens résidant à l'étranger, qu'il pousse à rentrer en leur proposant de financer leur séjour dans des universités équatoriennes. Cette deuxième étape du Plan voit aussi l'apparition d'un plan de retour spécifiquement destiné aux professeurs du primaire et du secondaire, mis en place par le ministère de l'Éducation. Celui-ci cherche à attirer des membres du corps enseignant partis exercer des métiers qui ne correspondent souvent pas à leurs qualifications, en leur proposant des avantages salariaux à leur retour. Le ministère de la Santé publique vise quant à lui un autre public: celui des professionnels de la santé, auxquels il propose de participer au plan Ecuador saludable, Voy por tí (Équateur sain, je viens à toi).

Il ne faut pourtant pas oublier que la majorité de ceux qui sont partis à la fin des années 1990 et au début des années 2000 sont des migrants peu qualifiés, qui n'ont généralement pas eu l'occasion de gagner en capital humain pendant leur migration, du fait du caractère extrêmement segmenté du marché de l'emploi dans les pays d'accueil et, partant, de leur cantonnement à des emplois très peu qualifiés. Force est de constater qu'il y a donc un décalage de plus en plus important entre les politiques mises en place et les besoins de la majorité des retornados.

37 À ce décalage entre la figure du retornado comme agent économique, qui domine dans les politiques publiques, et la vulnérabilité dans laquelle se retrouvent beaucoup des migrants à leur retour, vient s'ajouter un décalage entre l'aspect volontaire du retour, qui sous-tend ces politiques, et l'expérience beaucoup plus nuancée de ceux qui rentrent. En laissant de côté le cas des expulsés et des déportés, que le gouvernement ne considère bien évidemment pas comme des agents de leur retour, il me semble nécessaire de préciser que pour de nombreux migrants, le retour est une nécessité. Ces 
retours sont motivés par divers facteurs qui peuvent être d'ordre structurel ou beaucoup plus individuel. L'exemple le plus évident de ces retours contraints sont ceux déterminés par la crise économique, mais il ne faut pas négliger le poids que peuvent avoir des décisions prises à l'échelle familiale sur les projets migratoires. Bien des retours peuvent être vécus comme contraints, lorsqu'ils naissent de la nécessité de s'occuper de parents vieillissants ou d'enfants arrivés à l'adolescence par exemple.

La durabilité du retour, qui semble être une évidence pour les institutions chargées de le penser, doit également être remise en question, puisque les trajectoires des retornados sont loin d'être linéaires. Les projets migratoires de ces Équatoriens sont l'objet de renégociations constantes : si pour certains le retour était pensé dès le départ comme une simple stratégie de résistance face à la crise, donc nécessairement comme une situation temporaire, pour d'autres étant rentrés avec un projet à long terme, l'envie d'une nouvelle émigration peut surgir pour diverses raisons au bout de quelques mois ou de quelques années. Or les politiques pensées sous le gouvernement de Rafael Correa n'envisagent aucunement ces retours plus transitoires et ne prennent pas en compte les besoins spécifiques des circulants dont les identités sont beaucoup plus complexes que ne le laisse entendre le discours de Rafael Correa. S'il met en avant une identité monolithique qui pousse à penser le retour sur le modèle du retour d'Ulysse, c'est-à-dire comme l'étape finale d'une trajectoire douloureuse et comme un retour à l'état naturel des choses, les réalités des expériences sont bien plus complexes que cela.

Lorsque l'on regarde d'un peu plus près le Plan Bienvenid@s a casa, et plus spécifiquement les programmes destinés à faciliter le retour, force est de constater que l'impératif économique prend donc le pas sur la rhétorique des droits, notamment du droit de rentrer "de manière volontaire, digne et durable ${ }^{44}$. María Isabel Moncayo, qui le montre très bien dans sa recherche, va jusqu'à dire que le modèle de développement auquel font appel les politiques du retour ne font que perpétuer les écarts socio-économiques préexistant parmi les migrants ${ }^{45}$. Il me semble par ailleurs essentiel d'aller au-delà de la perspective économiciste du gouvernement, et de ne pas laisser de côté les aspects sociaux et culturels de la (ré)insertion des retornados. La migration de retour est bien plus qu'un simple déplacement spatial vers le pays d'origine, c'est un véritable processus qui nécessite que l'on se penche sur les recompositions des lieux d'ancrage et sur les formes d'habiter déployées par ces migrants dont le retour à la casa n'est pas non plus aussi linéaire que ne le postule Rafael Correa. Alors que la vision du « chez soi » émanant de la sphère institutionnelle repose avant tout sur l'idée d'enracinement, j'argue que celle des migrants est bien plus complexe et relationnelle. On en revient finalement à la confrontation entre des homophones chers aux chercheurs dans le champ des migration studies : on a d'un côté une vision de la casa fondée sur les roots (racines) - celle de l'État - et de l'autre une vision de la casa fondée sur les routes (chemins) - celle des migrants de retour. Pour comprendre la façon dont ces derniers habitent les lieux auxquels ils (re)viennent, il faut arriver à penser des géographies plus mobiles, multiples et transculturelles du «chez soi ». 


\section{NOTES}

1. "La mayor gratitud para nuestros hermanos migrantes [...] aquellos exiliados de la pobreza, expulsados de su propia tierra, supieron llevar la Patria pintada por dentro [...]; fueron los que nos mantuvieron con sus trabajos, con sus sacrificios, con sus remesas, aún a costa de la distancia y el desarraigo. Vuelvan queridos hermanos, [...] el Ecuador, su país, los espera con los brazos y el alma abiertos de par en par." Pour la citation complète, voir Rafael Correa, Posesión presidencial : El capital domina al mundo, discours prononcé les 24 mai 2013, disponible sur https:// www.presidencia.gob.ec/wp-content/uploads/downloads/2016/11/2013.05.24-DISCURSO-DEPOSESI \%C3 \%93N-MANDATO-2013-2017.docx, consulté le 16 novembre 2018.

2. Rafael Correa, Intervención del Presidente de la República, Rafael Correa, durante la posesión presidencial, discours prononcé le 10 août 2009, disponible sur http://www.presidencia.gob.ec/ wp-content/uploads/downloads/2013/10/10-10-09-Discurso_posesion_Presidencial.pdf, consulté le 14 décembre 2016.

3. Abdelmalek Sayad, L'Immigration ou les paradoxes de l'altérité. 1. L'illusion du provisoire, Paris, Raisons d'Agir Éditions, 2006, p. 188.

4. Steven Vertovec, "The Cultural Politics of Nation and Migration", Annual Review of Anthropology, $\mathrm{n}^{\circ} 40,2011$, p. 245.

5. L'Austro équatorien comprend principalement les provinces de Cañar et Azuay.

6. Brian Gratton, "Ecuadorians in the United States and Spain: History, Gender, and Niche Formation", Journal of Ethnic and Migration Studies, vol. 33, $\mathrm{n}^{\circ}$ 4, 2007.

7. Les chiffres exacts sont difficiles à obtenir puisqu'une grande partie de cette émigration s'est faite par des voies irrégulières, mais c'est le chiffre sur lequel la majorité des chercheurs spécialistes de la question sont d'accord. Voir, par exemple, Jacques Ramírez Gallegos, "A diez años de la estampida migratoria ecuatoriana: patrones y procesos de los flujos migratorios", in Jacques Ramírez (dir.), Con o sin pasaporte: Analisis socio-antropológico sobre la migración ecuatoriana, Quito, IAEN, 2010.

8. William Herrera Ríos, «S'emparer des “absents" : la construction du Secrétariat national du migrant de l'Équateur (2007-2013). Entre compétitions politiques, concurrences bureaucratiques et fragilités d'une innovation institutionnelle ", thèse soutenue sous la direction de M. Michel Dobry, Université Paris 1 Panthéon-Sorbonne, 2016.

9. Organisation internationale pour les migrations, "Termes clés de la migration ", disponible sur : https://www.iom.int/fr/termes-cles-de-la-migration, consulté le 15 novembre 2018.

10. Jacques Ramírez, Franklin Ramírez, La estampida migratoria ecuatoriana: crisis, redes transnacionales y repertorios de acción migratoria, Quito, Centro de Investigaciones CIUDAD UNESCO - Abya Yala, ALISEI, 2005.

11. Paolo Boccagni, "Making the 'Fifth Region' a Real Place? Emigrant Policies and the Emigration-Nation Nexus in Ecuador", National Identities, vol. 16, n 2, 2014, p. 117-137.

12. Alba Goycoechea, Franklin Ramírez, "Se fue, ¿a volver? Imaginarios, familia y redes sociales en la migración ecuatoriana a España (1997-2000)”, Íconos, nº 14, 2002, p. 32- 45.

13. "Es que uno llegó, y luego, así, todo el mundo fue saliendo. La facilidad era que España no necesitábamos visa, a Estados Unidos sí. En España, entrabamos como turistas [...] Como la gente ya veía que el uno pasa, el otro también, pues todo el mundo decía que: ‘Ay, que España esto, que España se gana dinero, que España todo...'. Pues, y todo el mundo se abrió los ojos, y todo el mundo salió”. Pour la citation complète, voir Amanda Bernal, « Migration et emploi : le cas des réémigrants latino-américains naturalisés espagnols résidant dans la capitale britannique ", mémoire de Master 2, sous la direction de M. Elies Furió Blasco et Mme Françoise Lestage, École Normale Supérieure de Lyon, 2015. 
14. Gioconda Herrera Mosquera, María Isabel Moncayo, Alexandra Escobar Garcia, Perfil Migratorio del Ecuador 2011, Quito \& Genève, Organisation internationale pour les migrations, 2012, p. 50.

15. Pour une comparaison détaillée des différentes politiques du retour à l'échelle de l'Amérique Latine, voir María Isabel Moncayo, "Las políticas de retorno en Sudamérica: Una ruta hacia el desarrollo?", in Gioconda Herrera (dir.), El vínculo entre migración y desarrollo a debate. Miradas desde Ecuador y América Latina, Quito, FLACSO - Ecuador, 2014, p. 155-181.

16. Ana Margheritis, "Todos Somos Migrantes (We Are All Migrants): The Paradoxes of Innovative State-led Transnationalism in Ecuador", International Political Sociology, vol. $5, \mathrm{n}^{\circ} 2$, 2011, p. 206.

17. "State-led transnationalism [...] refers to institutionalized national policies and programs that attempt to expand the scope of national state's political, economic, social, and moral regulation to include emigrants and their descendants outside the national territory", in Luin Goldring, "The Mexican State and Transmigrant Organizations: Negotiating the Boundaries of Membership and Participation", Latin American Research Review, 2002, vol. 37, n 3, p. 64.

18. William Herrera Ríos, op. cit.

19. Alberto Acosta, "El aporte de las remesas para la economía ecuatoriana", Expert Group Meeting on International Migration and development in Latin America and the Caribbean, Secrétariat des Nations Unies, Mexico, 30 novembre-2 décembre 2005.

20. Jacques Ramírez, La política migratoria en el Estado ecuatoriano. Rupturas, tensiones, continuidades y desafíos, Quito, Instituto de Altos Estudios Nacionales, 2013.

21. William Herrera Ríos, op. cit.

22. Paolo Boccagni, Jacques Ramírez, "Construyendo la Quinta Región: voto a distancia y análisis de la participación política de los ecuatorianos en el exterior”, in Jacques Ramírez (dir.), Con o sin pasaporte: Análisis socio-antropológico sobre la migración ecuatoriana, op. cit.

23. Ibid.

24. William Herrera Ríos, op. cit.

25. Ana Margheritis, op. cit.

26. "Defendemos un retorno entendido en sentido amplio: un retorno que no implica necesariamente el regreso físico, sino de forma más, la recuperación de las capacidades", citation présente à l'origine sur le site de la Secretaría Nacional del Migrante, www.senami.gov.ec, actuellement non-consultable mais cité in María Isabel Moncayo, El Plan "Bienvenid@s a casa": estudio sobre la experiencia del Fondo "El Cucayo", Madrid, Fundación Carolina - CeALCI, 2011.

27. Boccagni Paolo, op. cit.

28. Lisa Malkki, "National Geographic: The Rooting of Peoples and the Territorialization of National Identity Among Scholars and Refugees", Cultural Anthropology, n 7, 1, 1992, p. 24-44.

29. Pour une analyse plus détaillée, voir Chiara Pagnotta, "La identidad nacional ecuatoriana entre límites externos y internos", Amérique Latine Histoire et Mémoire. Les Cahiers ALHIM, n 16, 2008, disponible sur http://journals.openedition.org/alhim/3061, consulté le $1^{\mathrm{er}}$ janvier 2018.

30. "In Ecuador, exemplarily, the Fifth Region metaphor has been instrumental to a political discourse that celebrates the common grounds between stayers and leavers, in a simultaneously naturalistic and civic register, while downplaying the strong racial and ethnic divides within that national society", in Paolo Boccagni, op. cit.

31. J'utilise l'adjectif "révolutionnaire" dans un sens très restreint: il fait ici simplement référence au projet de Révolution Citoyenne autour duquel s'articule tout le programme politique de Rafael Correa.

32. Ces documents prendront dans un premier temps le nom de Plan Nacional de Desarrollo (Plan national de développement) puis de Plan Nacional para el Bien Vivir (Plan national pour le bien vivre). 
33. Secretaría Nacional del Migrante, Plan Nacional de Desarrollo Humano para las Migraciones 2007-2010, Quito, Gobierno Nacional de la República del Ecuador, 2007.

34. Heidi Armbruster, "Homes in Crisis: Syrian Orthodox Christians in Turkey and Germany", in Nadje Al-Ali, Khalid Koser (dir.), New Approaches to Migration? Transnational Communities and the Transformation of Home, Londres \& New York, Routledge, 2002, p. 20.

35. Je reprends le débat sur la définition de ce qu'est un «lieu» dans la géographie anglosaxonne. Voir Doreen Massey, "The Conceptualization of Place”, in Doreen Massey, Pat Jess (dir.), A Place in the World? Oxford, Oxford University Press in association with the Open University, 1995.

36. Arjun Appadurai, Modernity at Large: Cultural Dimensions of Globalization, Minneapolis, University of Minnesota Press, 1996, p. 192.

37. Henri Lefebvre, La Production de l'espace, Paris, Anthropos, 2000.

38. Pour une présentation et une analyse plus détaillée de ces politiques du retour, Voir Emmanuelle Sinardet, "La figura del migrante retornado a través de las políticas públicas: el caso ecuatoriano (2007-2014)", in Daniel Bengsch, Adriana Lopez Labourdette (dir.), Volver. Culturas e imaginarios del retorno a y desde América Latina, Barcelona, Linkgua, 2017.

39. Cucayo est un mot d'origine quichua qui fait référence aux provisions que l'on emporte avec soi lors d'un voyage. Pour plus de détails, voir Ronny Correa, Joan Lacomba Et Santiago Ochoa, "Crisis, retornos y emprendimientos. El caso de los migrantes ecuatorianos y el Fondo Cucayo", Revue européenne des migrations internationales, vol. 32, $\mathrm{n}^{\circ}$ 2, 2016, p. 95-119.

40. María Isabel Moncayo, El Plan "Bienvenid@s a casa": estudio sobre la experiencia del Fondo "El Cucayo", op. cit.

41. María Isabel Moncayo, "Las políticas de retorno en Sudamérica: ¿Una ruta hacia el desarrollo ?", op. cit.

42. Liliana Rivera Sánchez, "Narrativas de retorno y movilidad. Entre prácticas de involucramiento y espacialidades múltiples en la ciudad", Estudios Políticos, n 47, 2015.

43. María Isabel Moncayo, "Las políticas de retorno en Sudamérica: ¿Una ruta hacia el desarrollo?", op. cit.

44. Secretaría Nacional del Migrante, op. cit.

45. María Isabel Moncayo, "Migración y retorno en el Ecuador: entre el discurso político y la política de gobierno", mémoire de Master, sous la direction de Mme Gioconda Herrera, Quito, FLACSO - Ecuador, 2011.

\section{RÉSUMÉS}

À partir de la crise économique, politique et sociale de la fin des années 1990, l'Équateur connaît un mouvement d'émigration massive qui marquera durablement l'imaginaire collectif. Rafael Correa s'est emparé de cette question migratoire dès sa campagne présidentielle, en faisant de cette «tragédie nationale » une véritable ressource politique. Il continuera à l'exploiter au cours de ses mandats, puisqu'il fera de la question migratoire l'«étendard» de sa Révolution Citoyenne. C'est sur ce tournant institutionnel dans la façon dont l'état équatorien pense son lien à la diaspora que je me pencherai, en essayant de faire ressortir les limites de ces politiques présentées comme novatrices. 
A partir de la crisis económica, política y social que estalló en Ecuador a finales de los 90, se da un movimiento de emigración masiva que marcará duraderamente el imaginario colectivo. Rafael Correa supo apropiarse de dicha cuestión migratoria durante su campaña presidencial, y supo hacer de esta «tragedia nacional » un verdadero recurso político que siguió explotando al llegar al poder. Bajo sus gobiernos sucesivos, la cuestión migratoria se convertirá en el "estandarte" de su Revolución Ciudadana. En este artículo, analizaré el giro institucional en la manera en que el Estado ecuatoriano postula su vínculo con la diáspora, sin olvidar de subrayar los límites de un conjunto de políticas presentadas como novedosas.

\section{INDEX}

Mots-clés : migration, retour, Équateur, transnationalisme, chez-soi

Palabras claves : migración, retorno, Ecuador, transnacionalismo, casa

\section{AUTEUR}

\section{AMANDA BERNAL}

Université Paris Nanterre, CRIIA - EA 369/Aix-Marseille Université, TELEMME 